\title{
A55 PROTEOME ANALYSIS REVEALS AN UPREGULATION OF HEAT-SHOCK PROTEINS IN LPS-INDUCED PERITONEAL MACROPHAGES FROM MPGES-1 KNOCK-OUT MICE
}

M Korotkova, ${ }^{1}$ E Ossipova, ${ }^{1}$ H Idborg, ${ }^{1}$ K Ghorghe, ${ }^{1}$ P Leclerc, P-J Jakobsson' ${ }^{1}$ Rheumatology Unit, Department of Medicine, Karolinska Institutet, Stockholm, Sweden

\subsection{6/ard.2010.148965.25}

Background Microsomal prostaglandin E synthase-1 (mPGES-1) is the terminal enzyme in the induced $\mathrm{PGE}_{2}$ production at the sites of inflammation and well-recognised target for the development of novel anti-inflammatory drugs that can reduce symptoms of inflammation in rheumatic diseases. Genetic deletion of mPGES-1 in arthritic mice reduces inflammation, humoral immune response and protects them from pain and joint destruction. However, molecular mechanisms and signalling pathways involved in anti-inflammatory effects of selective mPGES-1 inhibition/deletion at sites of inflammation have not been explored.

Objective To study effects of mPGES-1 deletion on total proteins expression and eicosanoid profile upon lipopolysaccharide (LPS)-induced macrophage activation.

Methods Peritoneal macrophages (PM) from wild type (WT) and knock-out (mPGES-1-/-, knockout (KO)) mice were induced with LPS for $16 \mathrm{~h}$. Supernatants were harvested for eicosanoid analysis and proteins were isolated from the cells. Proteomics approach using high resolution mass spectrometry coupled to nanoflow-liquid chromatography has been employed to determine the identity and the relative abundance of expressed proteins. Eicosanoid profiling was performed using liquid chromatography-mass spectrometry.

Results Compared to wild-type, mPGES-1 deficient PM displayed a markedly attenuated increase in PGE2 production upon LPS stimulation, and exhibited increased levels of prostaglandin D2 metabolite and prostaglandin F2 $\alpha$. Comparative proteomic analysis of LPS-induced macrophages from WT and mPGES-1 KO mice identified 248 proteins with different molecular and cellular functions including cell death, cellular movement, cellular growth and proliferation, cellular function and maintenance and protein synthesis. In PM from KO mice, 109 proteins were upregulated, 101 proteins were downregulated and 38 proteins were not changed compared with PM from WT mice. Interestingly, among the top 10 proteins 
upregulated in macrophages from $\mathrm{KO}$ mice the authors identified two heat shock proteins with immune-modulatory properties, Hsp70-5 and Hsp70-8. Hsp70-5 (the immunoglobulin binding protein, $\mathrm{BiP}$ ) is known to be upregulated by cyclopentenone prostaglandins and suppress experimental arthritis in mice. Hsp70-8 (heat chock cognate 70, Hsc70) has been shown to modulate dendritic cells function. The expression of Hsp70-5 and Hsp70-8 in LPS-induced macrophages from WT and KO mice was confirmed by Western blot.

Conclusion The data reveal that inhibition of mPGES-1 activity in pro-inflammatory conditions resulted in the upregulation of heat-shock proteins with anti-inflammatory and immunomodulatory properties. 